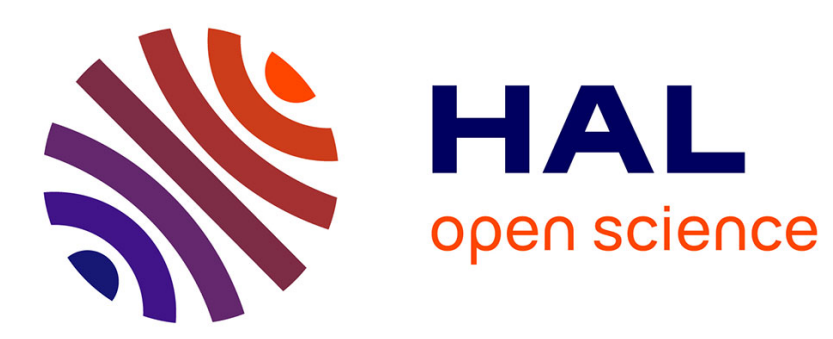

\title{
Reliable Multimedia Transmission in Wireless Ad-Hoc Networks for Telehealth Systems
}

Bernhard Hohmann, Merouane Debbah, Andreas Kröpfl

\section{To cite this version:}

Bernhard Hohmann, Merouane Debbah, Andreas Kröpfl. Reliable Multimedia Transmission in Wireless Ad-Hoc Networks for Telehealth Systems. 2ND INTERNATIONAL CONFERENCE ON PERVASIVE COMPUTING TECHNOLOGIES FOR HEALTHCARE 2008, Jan 2008, Finland. 4 p. hal-00329492

\section{HAL Id: hal-00329492 \\ https://hal-centralesupelec.archives-ouvertes.fr/hal-00329492}

Submitted on 10 Oct 2008

HAL is a multi-disciplinary open access archive for the deposit and dissemination of scientific research documents, whether they are published or not. The documents may come from teaching and research institutions in France or abroad, or from public or private research centers.
L'archive ouverte pluridisciplinaire HAL, est destinée au dépôt et à la diffusion de documents scientifiques de niveau recherche, publiés ou non, émanant des établissements d'enseignement et de recherche français ou étrangers, des laboratoires publics ou privés. 


\section{Reliable Multimedia Transmission in Wireless Ad-Hoc Networks for Telehealth Systems}

\author{
Bernhard Hohmann \\ Graz University of Technology, \\ Rechbauerstrasse 12, \\ 8010 Graz, Austria, \\ bernie@ sbox.tugraz.at
}

\author{
Mérouane Debbah \\ Supélec, Alcatel-Lucent Chair in flexible radio, \\ 3 rue Joliot-Curie, \\ 91192 Gif Sur Yvette Cedex, France, \\ merouane.debbah@supelec.fr
}

\author{
Andreas Kröpfl \\ Zydacron $\mathrm{GmbH}$, \\ Teslastrasse 4, \\ 8074 Grambach, Austria, \\ a.kroepfl@zydacron.com
}

\begin{abstract}
This paper proposes a time slotted distributed protocol to enhance transmissions in ad-hoc networks. This is achieved by employing the well known cooperative virtual multiple input multiple output (MIMO) technique. A general protocol for $N$ cooperating nodes is presented and evaluated considering bit error rate (BER), signal to noise ratio (SNR) and delay as target metrics. We show in particular that there is a trade off between performance, complexity and delay. Interestingly, reliable multimedia transmission can be achieved with reasonable delay constraints, as required in many telehealth applications. ${ }^{1}$
\end{abstract}

\section{INTRODUCTION}

Ad-hoc networks are gaining more and more importance, due to their flexibility and ease of deployment. However, the problem of fading is crucial in wireless communications and one needs to improve the reliability of these networks. One way of doing that is to apply diversity techniques such as multiple input multiple output (MIMO) [2]. These systems require, however, multiple antennas at the sender and/or at the receiver. If the nodes do not have multiple antennas due to cost or power constraints, there is the possibility to employ virtual MIMO or cooperative diversity schemes as shown for example in [3]. In this paper, we will focus our attention on two specific techniques of cooperation, namely amplify and forward (AF) and decode and forward (DF) [3]. In order to improve the performance of the DF scheme, we derive an extension of the Alamouti scheme that was introduced in [4]. This scheme is a simple diversity technique for two transmitting and one or two receiving antennas which achieves full diversity. This paper, however, extends the approach to a virtual Alamouti scheme with $N$ cooperating nodes. One key feature of this scheme is that only linear processing at the receiver is involved. This makes this scheme very attractive for receivers with power constraints. The primary application of the developed protocol is for telehealth applications which are becoming more and more important (see for example [5], [6]). One particular example of interest for the authors are homecare scenarios with distributed interactive terminals ${ }^{2}$ and monitoring cameras, where it is interesting to use adhoc networking. In such scenarios, multimedia data has to be transmitted reliably and the proposed scheme provides new

\footnotetext{
${ }^{1}$ This work is supported in part by the COAST [1] project group.

${ }^{2}$ See for example the Zydacron Carestation, www.zydacron.com
}

possibilities in the field. The paper is organized as follows. Section II introduces the system model and in Section III the protocol is described in detail. Section IV discusses simulation results and finally, Section V summarizes the paper with some concluding remarks.

\section{SySTEM MODEL}

In the proposed system model, $N$ nodes intend to cooperate in order to transmit data reliably. In the simulations, no coding delays are considered and the nodes are assumed to be synchronized. Furthermore, we consider a flat fading (which can be achieved by using an OFDM type technology) additive white gaussian noise (AWGN) channel which stays constant over at least one joint transmission of the participating nodes. The normalized transmit energy is fixed to 1 . The general channel model for a two way communication follows below, where $y$ is the received symbol, $x$ is the transmitted symbol, $h$ is the channel and $n$ is a zero mean gaussian noise with unit variance.

$$
y=h x+n
$$

A graph illustrating the cooperation between the nodes is given in Fig. 1. In the graph, $S, D$ and $R_{i}$ represent the sending node, the destination node and the relays, respectively. The naming of the corresponding channels is as well included in the graph, e.g. the channel from the second relay $R_{2}$ to the destination $D$ is called $h_{2 D}$. The total received signal at the destination $D$ for $N$ cooperating nodes (i.e. $N-1$ relays) for a DF scenario and without any time slotted protocol is given by

$$
y=h_{S D} x+\sum_{i=1}^{N-1} h_{i D} x+n
$$

(where the signal is decoded first at each relay and then forwarded). In this case, every relay first perfectly decodes $x$ and then forwards it toward the destination. In the AF case the received signal at the relay is simply forwarded. Thus, it forwards $h_{S i} x+n_{S i}$, which is the received signal at the relay $R_{i}$. Hence, also the noise $n_{S i}$ gets amplified and the total received signal at the destination $D$ is given by

$$
y=h_{S D} x+\left(\sum_{i=1}^{N-1} h_{i D} h_{S i} x+h_{i D} n_{S i}\right)+n
$$




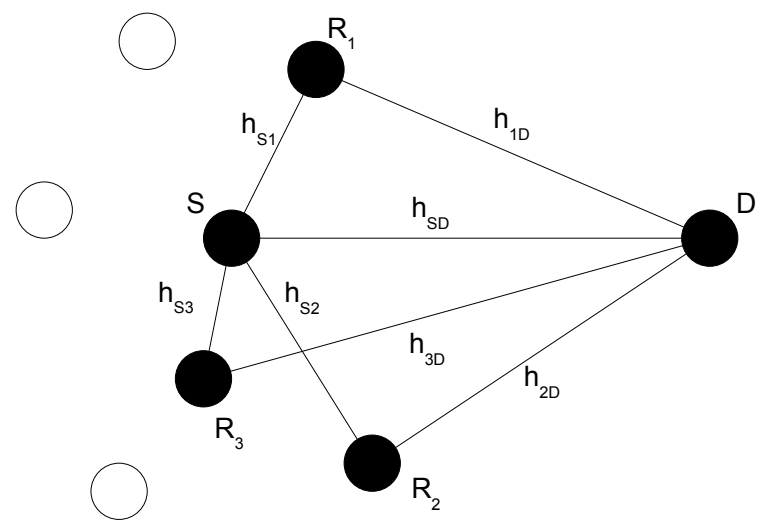

Fig. 1. System model, where $S, D$ and $R_{i}$ represent the sending node, the destination node and the relays, respectively. The channels are labeled according to the nodes they are connecting, thus, the channel from the second relay $R_{2}$ to the destination $D$ is called $h_{2 D}$.

TABLE I

TIME SLOTTED STRUCTURE FOR AMPLIFY AND FORWARD

\begin{tabular}{l|cccccc} 
& $t_{1}$ & $t_{2}$ & $t_{3}$ & $t_{4}$ & $\cdots$ & $t_{N}$ \\
\hline$S$ & $x_{1}$ & & & & & \\
$R_{1}$ & & $x_{1}$ & & & & \\
$R_{2}$ & & & $x_{1}$ & & & \\
$R_{3}$ & & & & $x_{1}$ & & \\
$\vdots$ & & & & & $\ddots$ & \\
$R_{N-1}$ & & & & & & $x_{1}$ \\
\hline$D$ & $y_{1}$ & $y_{2}$ & $y_{3}$ & $y_{4}$ & $\cdots$ & $y_{N}$
\end{tabular}

Unfortunately, as one can see, if all the relays send simultaneously, inter-symbol interference appears and the symbols can only be decoded by a computationally expensive detector. In order to avoid this, we apply a time slotted protocol.

\section{PRotocol}

The protocol is based on a time slotted structure where the sender picks a predefined number of $N-1$ participating relays out of the nodes within reach. As two different strategies are implemented, the protocol will be described in the following two sections, describing first the AF strategy in Section III-A and then DF in Section III-B.

\section{A. Amplify and Forward}

The AF strategy [3] is simple. In the first time slot the sender broadcasts a symbol to all the relays and in the following time slots all the relays transmit it one after the other. The symbols can then be decoded by a maximum likelihood (ML) decision, without inter-symbol interference for which the complexity is only related to the size of the constellation (and not the number of relays as previously). Thus, full diversity is achieved at the expense of a non-negligible delay. The time slotted structure is illustrated in Table I.

The quality of the received signal strongly depends on the energy of the channels to and from a relay, i.e., $\left|h_{S i}\right|^{2}$ and $\left|h_{i D}\right|^{2}$, as they are amplifying or attenuating the signal. Hence, the decision on which $N-1$ nodes are chosen as relays is
TABLE II

ALAMOUTI ENCODING AND TRANSMISSION SEQUENCE

\begin{tabular}{c|ccc} 
& $S$ & $R_{1}$ & $D$ \\
\hline$t_{3}$ & $x_{1}$ & $x_{2}$ & $y_{1}$ \\
$t_{4}$ & $-x_{2}^{*}$ & $x_{1}^{*}$ & $y_{2}$
\end{tabular}

based on these energies. In case of one relay, the optimal node is the one which maximizes the equation below, as it expresses the energy $E$, received at the destination.

$$
E=\frac{\left|h_{S i}\right|^{2}\left|h_{i D}\right|^{2}}{\sigma^{2}\left(1+\left|h_{i D}\right|^{2}\right)}
$$

where $\sigma$ is the variance of the noise. The optimal solution for choosing $N-1$ relays is straightforward and given by the following metric

$$
\begin{aligned}
E=\frac{\left|h_{S i}\right|^{2}\left|h_{i D}\right|^{2}+\left|h_{S(i+1)}\right|^{2}\left|h_{(i+1) D}\right|^{2}+\cdots}{\sigma^{2}\left((N-1)+\left|h_{i D}\right|^{2}+\left|h_{(i+1) D}\right|^{2}+\cdots\right.} \\
\\
\frac{\cdots+\left|h_{S(N-1)}\right|^{2}\left|h_{(N-1) D}\right|^{2}}{\left.\cdots+\left|h_{(N-1) D}\right|^{2}\right)}
\end{aligned}
$$

In this formula, all possible combinations of the available set of nodes have to be considered. This would be computationally very expensive. Thus, all available nodes are one after another set in Equation 4 and ranked by $E$. After that, the best $N-1$ nodes are chosen in the order of maximizing the equation, which still gives good results.

\section{B. Decode and Forward}

In case of DF, the sender $S$ broadcasts two symbols $x_{1}$ and $x_{2}$ to an odd number $(N-1)$ of relays in the first two time slots $t_{1}$ and $t_{2}$. Then the relaying nodes first have to decode the received signal and then reencode it. Let us first consider a simple example of one relay $R_{1}$ helping the source $S$ to transmit these symbols. Applying the Alamouti scheme [4] here, the symbols have to be encoded according to Table II, in time slots $t_{3}$ and $t_{4}$. The destination node now has just to perform a linear combination of the two received symbols $y_{1}$ and $y_{2}$ and the channels $h_{1 D}$ and $h_{S D}$, which is computationally inexpensive and, thus, energy efficient. The linear combinations follow below

$$
\begin{aligned}
& \hat{y}_{1}=h_{S D}^{*} y_{1}+h_{1 D} y_{2}^{*}=x_{1}\left(\left|h_{1 D}\right|^{2}+\left|h_{S D}\right|^{2}\right)+n \\
& \hat{y}_{2}=h_{1 D}^{*} y_{1}-h_{S D} y_{2}^{*}=x_{2}\left(\left|h_{1 D}\right|^{2}+\left|h_{S D}\right|^{2}\right)+n
\end{aligned}
$$

The linear combinations $\hat{y}_{1}$ and $\hat{y}_{2}$ give an amplified version of the original symbols $x_{1}$ and $x_{2}$ respectively. By a ML decoding the estimates of $x_{1}$ and $x_{2}$ can be retrieved. It has to be noted that perfect channel knowledge is assumed. In case of more than one relay, the time slotted structure which is shown in Table III is applied. The initial steps (time slots $\left.t_{1}, \ldots, t_{4}\right)$ are the same as if only one relay was used. In the following time slots, pairs of two relays consecutively transmit the two symbols in the same manner until all the nodes have transmitted the same symbols. As the symbols are sent over 
TABLE III

TIME SLOTTED STRUCTURE FOR DECODE AND FORWARD

\begin{tabular}{l|ccccccccc} 
& $t_{1}$ & $t_{2}$ & $t_{3}$ & $t_{4}$ & $t_{5}$ & $t_{6}$ & $\cdots$ & $t_{N+1}$ & $t_{N+2}$ \\
\hline$S$ & $x_{1}$ & $x_{2}$ & $x_{1}$ & $-x_{2}^{*}$ & & & & & \\
$R_{1}$ & & & $x_{2}$ & $x_{1}^{*}$ & & & & & \\
$R_{2}$ & & & & & $x_{1}$ & $-x_{2}^{*}$ & & & \\
$R_{3}$ & & & & & $x_{2}$ & $x_{1}^{*}$ & & & \\
$\vdots$ & & & & & & & $\ddots$ & & \\
$R_{N-2}$ & & & & & & & $\ddots$ & $x_{1}$ & $-x_{2}^{*}$ \\
$R_{N-1}$ & & & & & & & & $x_{2}$ & $x_{1}^{*}$ \\
\hline$D$ & $y_{1}$ & $y_{2}$ & $y_{3}$ & $y_{4}$ & $y_{5}$ & $y_{6}$ & $\cdots$ & $y_{N+1}$ & $y_{N+2}$
\end{tabular}

different routes, a diversity gain to overcome deep fades is achieved.

The strategy of choosing the relay nodes is slightly simpler here. In this scheme we assume that it is sufficient to consider the energy of the channel between sender and relay, i.e., $\left|h_{S i}\right|^{2}$. This channel is of course more important than the channel between relay and destination, as the relay has to reliably decode the symbol. Otherwise the relay could forward a wrong symbol and, thus, degrade the joint transmission. Therefore, nodes are simply chosen in order of their channel energies $\left|h_{S i}\right|^{2}$.

\section{ANALYSis}

Simulations have been performed for BPSK and QPSK. The performance of $\mathrm{AF}$ and $\mathrm{DF}$ in terms of BER vs. SNR is analyzed. Furthermore, the delay and the node selection strategy are evaluated.

\section{A. BER vs. SNR}

The results for AF and DF using BPSK encoding are presented in Fig. 2 and Fig. 3. It can be observed, that in the two node scenario, i.e., one relay, $\mathrm{AF}$ is performing better than DF. This is due to the optimum relay selection technique of AF in case of one relay. However, employing more relays, DF outperforms AF significantly. For example, DF with 6 nodes gives better results than $\mathrm{AF}$ with 8 nodes over the whole simulated SNR range. Furthermore, in low SNR regions DF performs better than AF at any number of nodes.

In case of QPSK, shown in Fig. 4 and Fig. 5, the advantage of DF over AF increases. Here, the supremacy of AF in case of one relay becomes vanishingly small. Furthermore, DF with 4 nodes outperforms the 8 node AF simulation. However, much more nodes have to cooperate, to achieve low BERs at a given SNR, when employing QPSK constellations.

\section{B. Delay}

By increasing the number of cooperating nodes the performance increases likewise. Theoretically, it would be possible to reach almost any BER at a given SNR. This, however, is in most cases very unrealistic, because the delay grows as well significantly. Due to space constraints, but without loss of concision, only BPSK results are presented for the delay and node selection performance analysis. In Fig. 6, the delay for BPSK in time slots vs. BER at SNR $=8$ is presented. In $\mathrm{DF}$, in one cooperative transmission always two symbols are

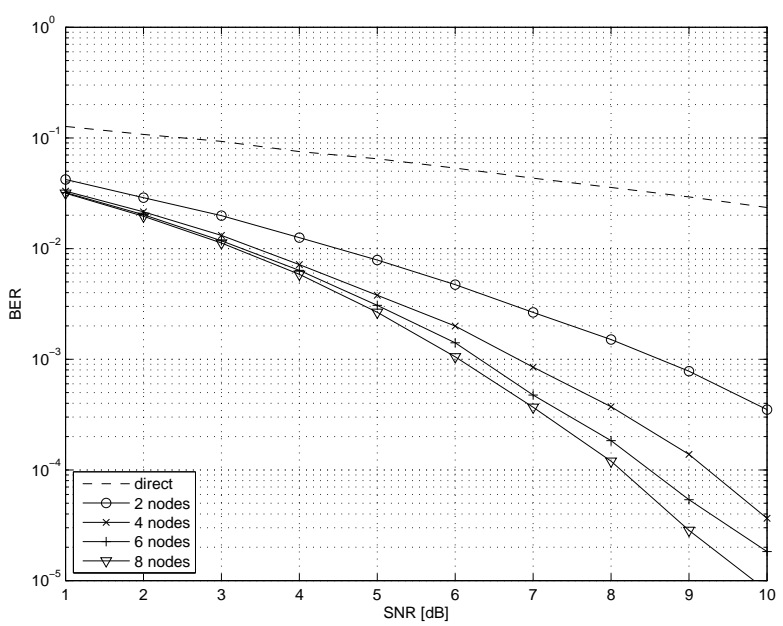

Fig. 2. The BER vs. SNR for AF (BPSK).

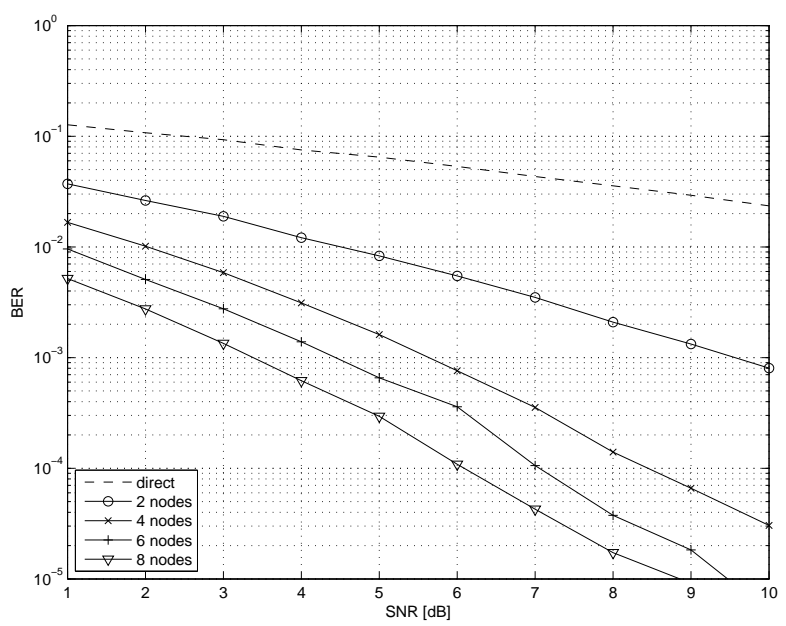

Fig. 3. The BER vs. SNR for DF (BPSK).

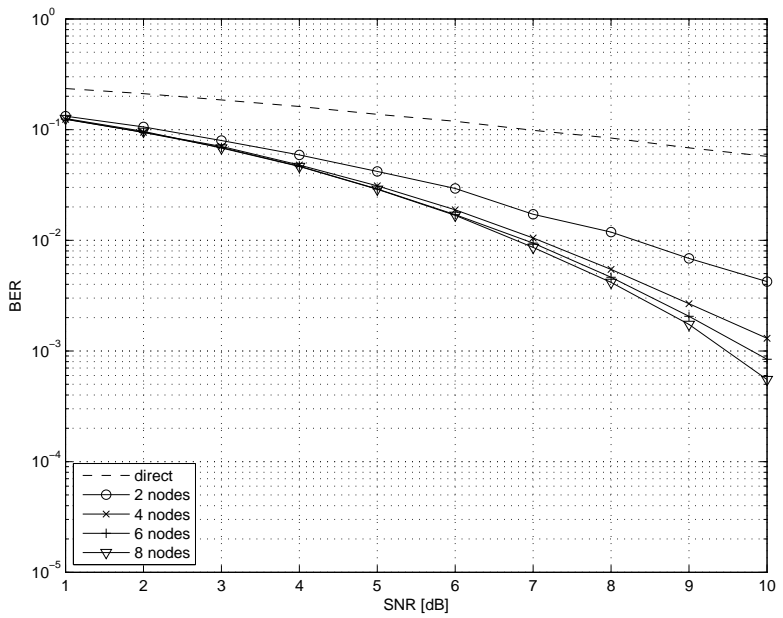

Fig. 4. The BER vs. SNR for AF (QPSK). 


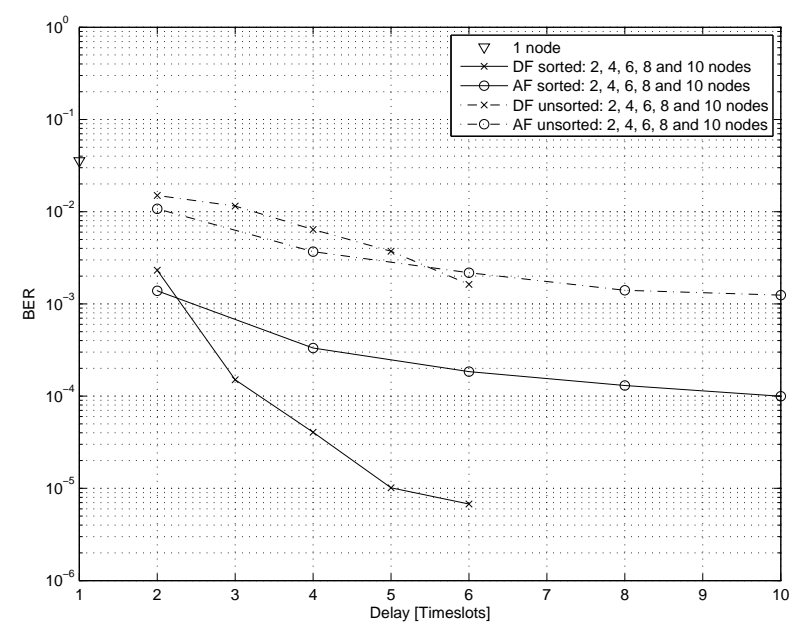

Fig. 6. The delay in time slots vs. BER for AF and DF using BPSK encoding at $\mathrm{SNR}=8$. The dash dotted lines show the curves obtained with the same input parameters, but without any node selection strategy.

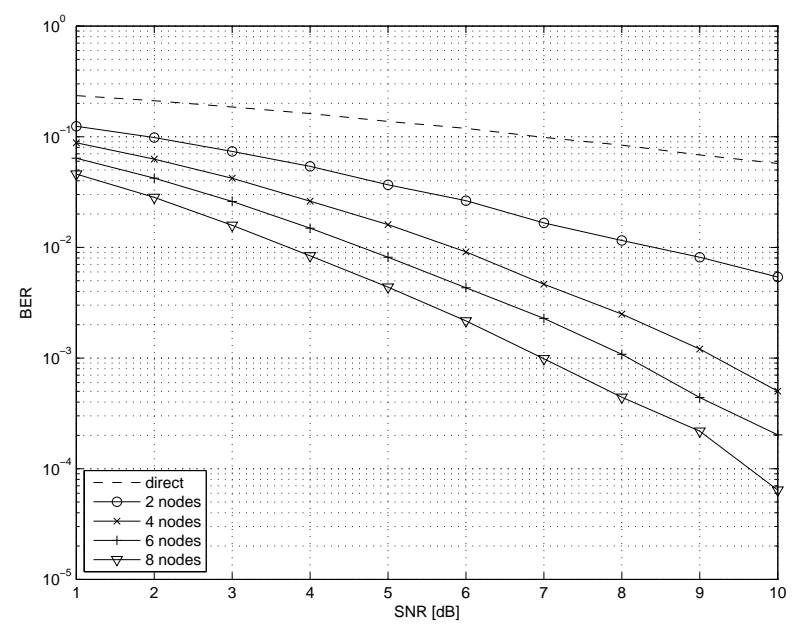

Fig. 5. The BER vs. SNR for DF (QPSK).

transmitted jointly due to the coding scheme. In contrast to AF where always just one symbol is treated. In order to be able to compare AF and DF, the delay in time slots is drawn for the transmission of one symbol for both AF and DF. It can be observed that DF performs better in means of delay. DF is faster and reaches much better BER, except for the two node scenario, where AF and DF have the same delay of two time slots. The superiority of DF over AF becomes again much more obvious with QPSK. Here the BER curve flattens out very fast for AF, whereas for DF it still steadily decreases at 10 nodes. However, coding delays which have higher impact on DF than AF are not considered. It is interesting to note that the DF curve with BPSK constellations seems to start flattening out at 10 nodes as it is already in a low BER region of $10^{-5}$, whereas the DF curve in QPSK is still quite steep and only in the $10^{-3}$ BER region. This indicates that there might be a potential if one wants to use a great number of relays with higher order M-PSK constellations.

\section{Node Selection}

In order to prove the efficiency of the employed node selection strategy by simulation, a comparison with random node selection has been performed. In Fig. 6 the dash dotted graphs show the BER curves for random node selection. It can be observed that the node selection strategies for AF and DF result in a significant gain. Furthermore, the result of the optimal node selection strategy for AF in case of one relay can be observed when using BPSK. When employing QPSK constellations, the optimal node selection strategy for one relay equals out with the generally better performance of DF. Thus, the curves start at the same point.

\section{CONCLUSiON}

In this paper we presented a new scheme for cooperative transmission in ad-hoc networks which achieves full diversity. It is shown that by employing the well known Alamouti scheme in DF manner or even only an AF strategy, with just one relay the performance can be improved significantly. In this case AF might be preferable due to its simple implementation structure. However, the good performance in this case results mainly from the complex node selection strategy where all channels have to be known. The node selection strategy for DF on the other hand relies just on the knowledge of the channels from the sender to the relays, so it might be more realistic to implement. When employing more than just one relay, DF gives better BER performance at lower delays. Still, the delay increases linearly with the number of relays and, thus, high numbers of relays would usually not be preferable. Hence, in case of power constraints and high reliability demands, the proposed virtual MIMO protocol is shown to be the right approach. Future work will be on extending the scheme by employing other space-time block codes [7].

\section{REFERENCES}

[1] COAST, "www.coast.at."

[2] I. E. Telatar, "Capacity of multi-antenna gaussian channels," in European Transactions on Telecommunications, 1999, pp. 585-595.

[3] J. N. Laneman, D. N. C. Tse, and G. W. Wornell, "Cooperative diversity in wireless networks: Efficient protocols and outage behavior," in IEEE Transactions on Information Theory, Vol. 50, No. 12, 2004.

[4] S. M. Alamouti, "A simple transmit diversity technique for wireless communications," in IEEE Journal on Select Areas in Communications, Vol. 16, No. 8, 1998, pp. 1451-1458.

[5] A. Keshavarz, A. M. Tabar, and H. Aghajan, "Distributed vision-based reasoning for smart home care," Workshop on Distributed Smart Cameras, 2006.

[6] A. Williams, D. Xie, S. Ou, R. Grupen, A. Hanson, and E. Riseman, "Distributed smart cameras for aging in place," Workshop on Distributed Smart Cameras, 2006.

[7] V. Tarokh, H. Jafarkhani, and A. R. Calderbank, "Space-time block codes from orthogonal designs," in IEEE Transactions on Information Theory, Vol. 45, No. 5, 1999, pp. 1456-1467. 